\title{
Increased PD-L1 expression in erlotinib-resistant NSCLC cells with MET gene amplification is reversed upon MET-TKI treatment
}

\author{
Christina Demuth ${ }^{1}$, Morten Nørgaard Andersen ${ }^{1}$, Kristine Raaby Jakobsen ${ }^{1,2}$, Anne \\ Tranberg Madsen ${ }^{1}$ and Boe Sandahl Sørensen ${ }^{1}$ \\ ${ }^{1}$ Department of Clinical Biochemistry, Aarhus University Hospital, DK-8200 Aarhus N, Denmark \\ ${ }^{2}$ Department of Biomedicine, Aarhus University, DK-8000 Aarhus C, Denmark \\ Correspondence to: Christina Demuth, email: Demuth@clin.au.dk
}

Keywords: erlotinib, resistance, NSCLC, PD-L1, MET

Received: June 01, 2017 Accepted: July 25, $2017 \quad$ Published: August 04, 2017

Copyright: Demuth et al. This is an open-access article distributed under the terms of the Creative Commons Attribution License 3.0 (CC BY 3.0), which permits unrestricted use, distribution, and reproduction in any medium, provided the original author and source are credited.

\section{ABSTRACT}

Introduction: Cancer cells can achieve immune evasion by expressing the programmed death receptor 1 ligand (PD-L1) on the cell surface. Blockade of the receptor (PD-1) can avert this evasion. Here we aim at investigating PD-L1 expression in erlotinib-resistant lung cancer cells with MET proto-oncogene (MET) gene amplification.

Materials and Methods: We employed an erlotinib-resistant NSCLC cell line with MET gene amplification. PD-L1 mRNA (qPCR) and protein (flow cytometry) expression was investigated after treatment with MET and mitogen-activated protein kinase (MAPK) targeting drugs (crizotinib and SCH772984, respectively).

Results: We demonstrate that PD-L1 expression is increased in erlotinib-resistant non-small cell lung cancer (NSCLC) cells with MET gene amplification. Targeted inhibition of MET significantly decreases both gene and protein expression of PD-L1. Further, we demonstrate that inhibiting MAPK also results in a significant decrease in PD-L1 expression. Taken together these results show that expression of PD-L1 in the erlotinib-resistant cell line is associated with MET activity, and the downstream MAPK pathway.

Conclusions: Our results demonstrate that PD-L1 expression is increased in erlotinib resistant NSCLC cells with MET gene amplification and that the increase can be averted by targeted inhibition of MET.

\section{INTRODUCTION}

Worldwide, lung cancer is one of the most frequent cancers and mortality is high. The vast majority of lung cancers are non-small cell lung cancers (NSCLC). Over the past decade several oncogenic drivers have been identified [1], though most distinct are the observations of activating mutations in the epidermal growth factor receptor $(E G F R)$ of patients with adenocarcinoma histology. Today, treatment with EGFR-targeting tyrosine kinase inhibitors (TKIs), like gefitinib and erlotinib, is part of the routine treatment of patients harboring these mutations. This change in treatment has markedly increased the survival in this subgroup of patients [2], though treatment resistance is inescapable [3]. Several resistance mechanisms have been found and thoroughly described including the EGFR T790M mutation, MET proto-oncogene (MET) gene amplification, development of epithelial to mesenchymal transition (EMT), and transition to a small cell lung cancer (SCLC) phenotype [4].

Treatment of lung cancer with immunotherapy has gained much attention after promising clinical trials. Especially blockade of the pathway activated by the programmed death receptor 1 (PD-1) and its ligand PD-L1 is being massively investigated. PD- 1 is expressed on the surface of T-cells, and upon activation by the ligand, PD-1 initiates inactivation of the cell [5]. Expression of PD-L1 
on the surface of cancer cells leads to this inactivation of T-cells and helps the cancer cells achieve immune evasion [6]. The T-cell inactivating interaction can be inhibited by anti-PD-1 or anti-PD-L1 agents, and hereby antitumor immune activity can be restored [7, 8]. At present, no validated biomarker for response to PD-1-PD-L1 blockade has been presented [9], though studies suggest that expression of PD-L1 in tumor specimens could be a candidate biomarker $[10,11]$.

Studies of PD-L1 expression have shown an association with activating EGFR mutations in NSCLC [12-14]. Though, contradicting results have also been presented [15]. Activation of EGFR increases the expression of $\mathrm{PD}-\mathrm{L} 1$ in vitro through the mitogen-activated protein kinase (MAPK) pathway, and inhibition of EGFR using a TKI correspondingly decreases expression of PD-L1 [16-18]. This regulatory mechanism has been confirmed in vivo [19]. Despite increasing interest in the mechanisms of resistance to EGFR-TKIs, only few studies have engaged in investigating the expression of PD-L1, when resistance has emerged. In vitro data suggest that the T790M resistance mutation is accompanied by increased expression of $P D-L 1$ [16], and a retrospective clinical study finds association between gefitinib resistance and increased PD-L1 expression in a patient cohort [20]. If the dynamics of PD-L1 are affected by treatment and development of resistance, the timing of retrieving the biopsy used for investigating PD-L1 expression is of great importance.

The aim of this study was to investigate expression of $P D-L 1$ in erlotinib-resistant cells. The cells acquired resistance through $M E T$ gene amplification, and we further wanted to investigate if targeting the new oncogenic driver and the downstream proliferative pathway would affect the $P D-L 1$ expression.

\section{RESULTS}

\section{PD-L1 expression in erlotinib-resistant cells}

The erlotinib-resistant cell line HCC827ER was generated over approximately 4 months where the erlotinib concentration was gradually increased to a maximal concentration of $5 \mu \mathrm{M}$. A full description of the resistant cells is presented in Jakobsen et al. [21].

We investigated the gene expression of $M E T$ and $P D-L 1$ by qPCR at each erlotinib concentration during the establishment of HCC827ER. We observed that MET gene expression initially decreased but started increasing at approximately $200 \mathrm{nM}$ (see Figure 1A). At resistance the $M E T$ gene expression markedly surpassed that of the parental cell line. This increase followed the increase in gene copy number [21]. We observed a small decrease in $M E T$ gene expression at 3 and $4 \mu \mathrm{M}$ erlotinib, although it still greatly surpassed that of the parental cell line. Interestingly, the gene expression of $P D-L 1$ seemed to follow that of $M E T$. $P D-L 1$ gene expression was markedly decreased at the initiation of erlotinib treatment (see Figure 1B), but when the erlotinib concentration reached approximately $200 \mathrm{nM}$, expression of $P D-L 1$ started increasing and at the point of resistance $(5 \mu \mathrm{M}), P D$ $L 1$ expression had exceeded the expression level of the parental cells.

We used flow cytometry to investigate the expression of PD-L1 protein on the surface of the cells. As can be seen from Figure 1C, the median fluorescence intensity (MFI) and histograms were similar between the parental HCC827PAR and the HCC827ER cell line. These results corroborated the findings of increased $P D-L 1$ gene expression.

\section{PD-L1 expression was decreased in HCC827ER upon treatment with crizotinib}

Previous studies indicate a general link between receptor tyrosine kinase (RTK) activity and expression of $P D-L 1$ in erlotinib sensitive cells $[16,17,22]$. Our first results suggested that an association between increase in $M E T$ and $P D-L 1$ gene expression was also present in erlotinib-resistant cells. We therefore wanted to investigate if inhibition of MET, using a MET-targeting TKI (crizotinib), affected the expression of $P D-L 1$ in these cells.

The viability of HCC827ER cells following treatment with crizotinib has previously been determined [21]. We found that treatment with 0.1 and $1 \mu \mathrm{M}$ crizotinib sustained reasonable viability, while sufficiently inhibiting phosphorylation of MET (Figure 2A). A decrease in totalMET was also observed, though not as distinct as the decrease in phospho-MET. Furthermore, the downstream phosphorylation of Akt and MAPK was also decreased by crizotinib (see Figure 2A). Treatment with both 0.1 and $1 \mu \mathrm{M}$ crizotinib significantly decreased gene and protein expression of PD-L1 (Figure 2B) as measured by qPCR and flow cytometry.

$5 \mu \mathrm{M}$ erlotinib showed a tendency towards decreasing PD-L1 gene expression (data not shown), but this effect was not significant and neither was the trend seen on the protein expression (see Supplementary Figure 2). Combined treatment using both crizotinib and erlotinib had no additional effect on either cell viability or PD-L1 expression (data not shown).

These results demonstrated that inhibiting MET using crizotinib resulted in decreased PD-L1 expression.

\section{PD-L1 expression is decreased upon MAPK inhibition}

Since the MAPK pathway was activated by the MET gene amplification, and we saw that crizotinib inhibited phosphorylation of MAPK, we wanted to investigate if PD-L1 expression in HCC827ER was dependent on the MAPK pathway. Cells were treated with the MAPK inhibitor SCH772984. Cell viability was only mildly 
affected by the inhibitor at concentrations up to $5 \mu \mathrm{M}$ (see Figure 2C). Different concentrations of SCH772984 were tested and we found that 0.1 and $1 \mu \mathrm{M} \mathrm{SCH772984}$ was sufficient for decreasing MAPK activity (see Figure 2D). Total and activated Akt were stable or only weakly increased upon treatment.

After exposure to SCH772984 the HCC827ER cell line significantly decreased expression of $P D-L 1$ mRNA (see Figure 2E), as measured by qPCR. Further, PD-L1 cell surface expression was investigated by flow cytometry, and we found PD-L1 to be significantly decreased in treated cells as compared to the untreated control (see Figure 2E).

These results indicated that inhibiting MAPK led to decreased levels of PD-L1.

\section{PD-L1 expression in HCC827ER subclones}

The erlotinib resistant cell line can be divided into either MET-amplified or EMT subclones [21]. This polyclonality was also visualized in the flow cytometry histogram, where the HCC827ER cell line presented with a broad histogram and a tendency of two peaks, indicating clonal populations with either high or low expression of PD-L1 (Supplementary Figure 2).

We wanted to investigate how PD-L1 expression differed in these clonal subtypes. We measured $P D-L 1$ gene expression in 14 sub clones derived from HCC827ER (Figure $3 \mathrm{~A})$. There was a clear distinction between the two clonal subtypes; $M E T$-amplified clones generally presented with higher $P D-L 1$ expression than the EMT clones. Further, we

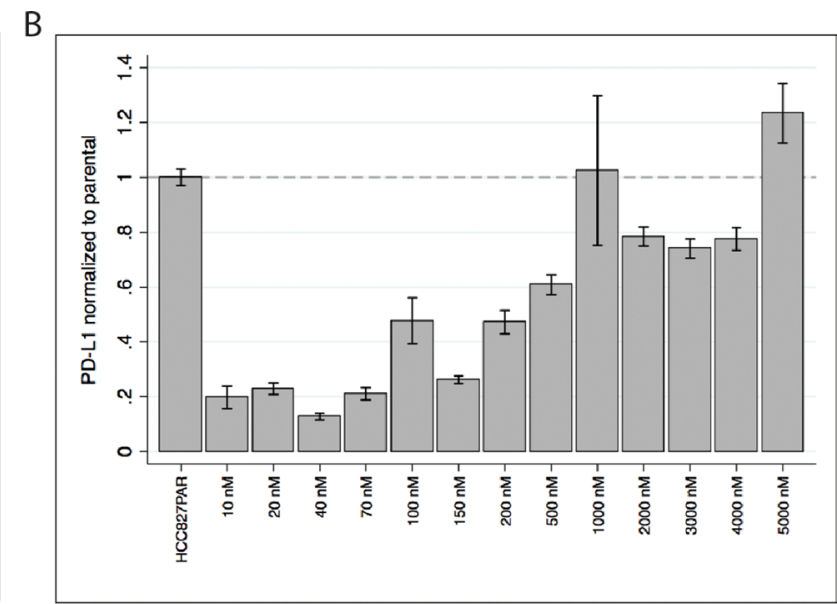

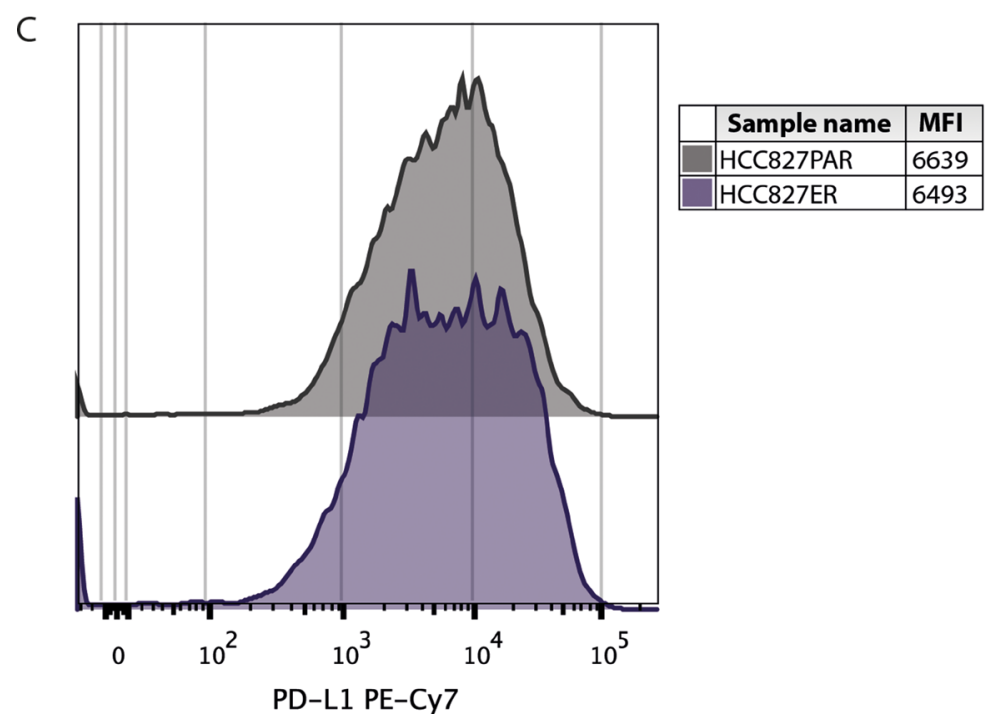

Figure 1: PD-L1 and MET gene expression and PD-L1 cell surface expression in HCC827ER. (A) MET gene expression was measured at each concentration-point during establishment of the resistant cell line (the erlotinib concentrations are indicated at the $\mathrm{x}$-axis), and normalized to the expression in the HCC827PAR cell line. Initially expression is decreased, but during resistance development expression starts increasing (approximately from $200 \mathrm{nM}$ erlotinib), and markedly exceeds that level of the HCC827PAR when resistance is established. (B) Correspondingly, $P D-L 1$ gene expression was measured during the resistance-development, and normalized to HCC 827PAR. PD-L1 expression also starts increasing at approximately $200 \mathrm{nM}$ erlotinib, and expression in the final erlotinib-resistant cell line exceeds that of HCC827PAR. (C) Using flow cytometry the PD-L1 protein expression was measured. A representative histogram and mean fluorescence intensity (MFI) values are presented. 
investigated the protein expression using flow cytometry on four of the 14 clones (two MET and two EMT clones, Figure $3 \mathrm{~B}$ and $3 \mathrm{C}$ ). The $M E T$-amplified clones (clone 2 and 3) had a pronounced increase in PD-L1 protein expression as compared to the original HCC827ER cell line, while the opposite was the case for the EMT clones (clone 4 and 10).

These results further strengthened the hypothesis of a correlation between MET activity and increased PD-L1
A

\begin{tabular}{|c|c|c|c|c|}
\hline $\begin{array}{l}\text { Erlotinib }(\mu M) \\
\text { Crizotinib }(\mu M)\end{array}$ & $\begin{array}{l}0 \\
0\end{array}$ & $\begin{array}{l}5 \\
0\end{array}$ & $\begin{array}{c}0 \\
0.1\end{array}$ & $\begin{array}{l}0 \\
1\end{array}$ \\
\hline Total-MET & - & {[} & - & $\ldots$ \\
\hline Phospho-MET & 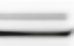 & 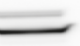 & - & \\
\hline Total-Akt & - & - & - & 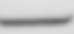 \\
\hline Phospho-Akt & 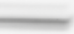 & {[} & & 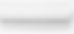 \\
\hline Total-MAPK & - & 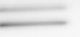 & 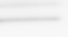 & - \\
\hline Phospho-MAPK & $=$ & $=$ & - & - \\
\hline H3 & - & - & - & $=$ \\
\hline
\end{tabular}

C
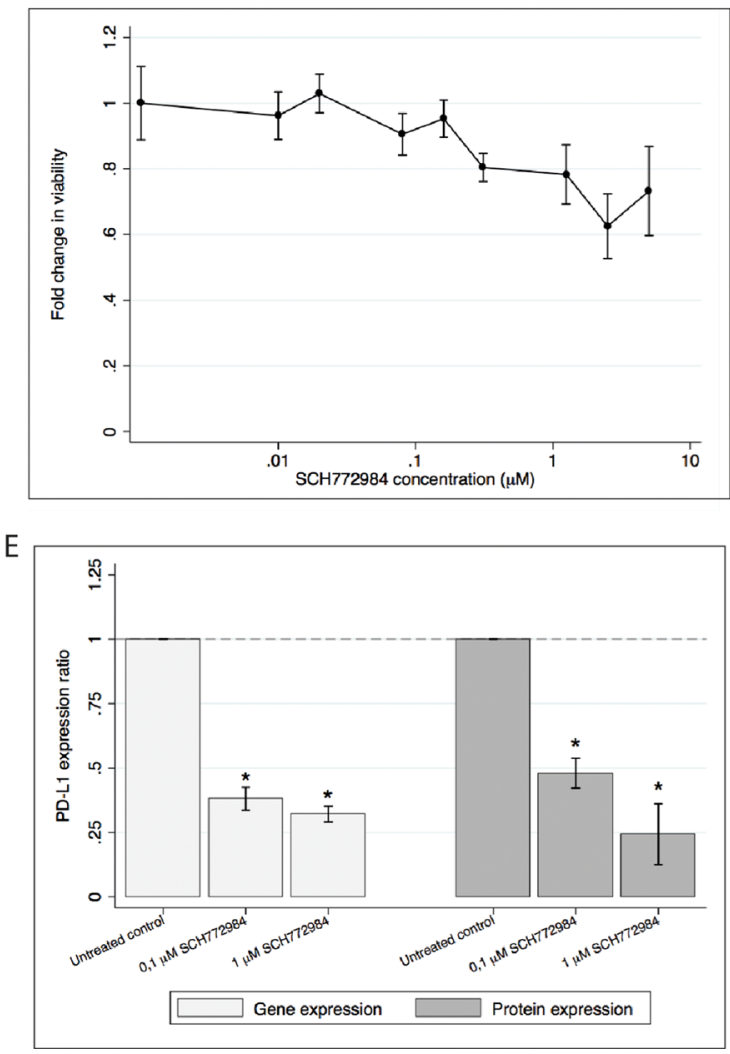

B
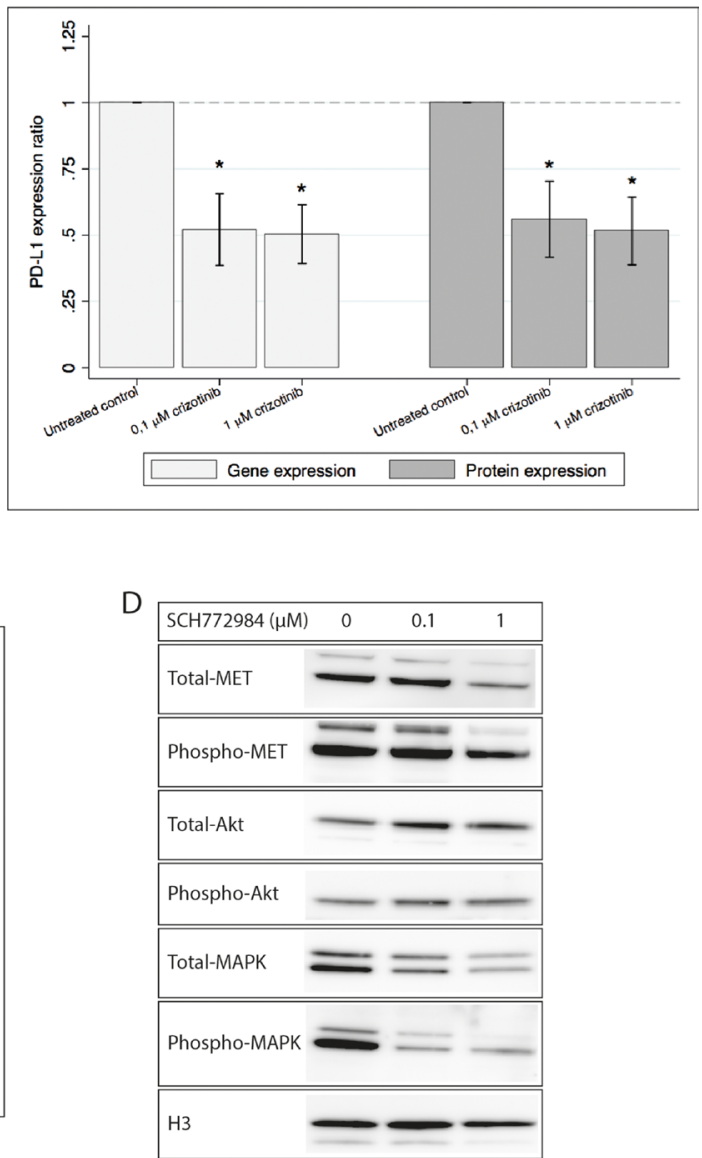

Figure 2: PD-L1 gene and protein expression is decreased in HCCC827ER upon treatment with either crizotinib or SCH779428. H3 is used as loading control in both western blots. All gene and protein expressions are normalized to an untreated control and presented as mean \pm SD. Gene and cell surface protein expression data is presented as mean of three individual experiments. * denotes significant difference $(p$-value < 0.05) (A) Western blot showing decreased activation of MET, Akt and MAPK after treatment with crizotinib, while erlotinib shows only a minor effect on MAPK. (B) PD-L1 gene and protein expression after treatment with crizotinib. Treatment with crizotinib significantly decreases the expression of PD-L1. (C) Cell viability after treatment with SCH772984. Viability measurements are normalized to an untreated control, and the fold change is plotted. (D) Western blot showing decreased MET, Akt and MAPK activity after treatment with SCH772984. (E) PD-L1 gene and protein expression following treatment with SCH772984. Gene expression and protein expression is significantly decreased by treatment with SCH772984. 
expression, while PD-L1 expression seemed to be lowered in EMT clones.

\section{DISCUSSION}

Immune evasion is one of the hallmarks of cancer [23]. One way of evading the immune system is by expressing PD-L1 on the surface of the cancer cell. This evasion can be targeted by immunotherapy, which is currently among the most promising new treatments of NSCLC. As of today the primary suggested biomarker for response to PD1/PD-L1 system blockade is expression of PD-L1. Further, studies have correlated expression of PD-L1 to EGFR mutational status and in vitro data have confirmed a regulatory function of EGFR [16, 17]. Though, not much is known about the dynamics in PDL1 expression when EGFR-TKI resistance occurs. In the present study, we have investigated expression of PD-L1 during development of erlotinib-resistance in the NSCLC cell line HCC827. The resistant cell line was generated using a clinically relevant erlotinib concentration [24]. We found that the expression is diminished initially, but levels exceed expression in the parental cell line, when resistance has developed. The resistant cells gain gene expression of the new oncogenic driver $M E T$ and we further demonstrate that expression of PD-L1 increases as expression of $M E T$ increases. Treatment with the MET-directed TKI crizotinib decreases expression of $P D-L 1$. Further, targeting MAPK, downstream in the signaling pathway of MET, also effectively decreases expression of PD-L1. These results suggest an association between MET activity and PD-L1 expression.

Our results are in accordance with previous findings of RTK regulated PD-L1 expression and its involvement with MAPK [16-18, 22, 25]. Taken together, these studies indicate a general regulatory mechanism of RTKs through the MAPK pathway, and in some cases, through the PI3K/ Akt pathway or a combination of the two [22, 26]. If PD-L1 expression is validated as a biomarker for PD1PD-L1 axis blockade, this makes TKI-resistance mediated
A

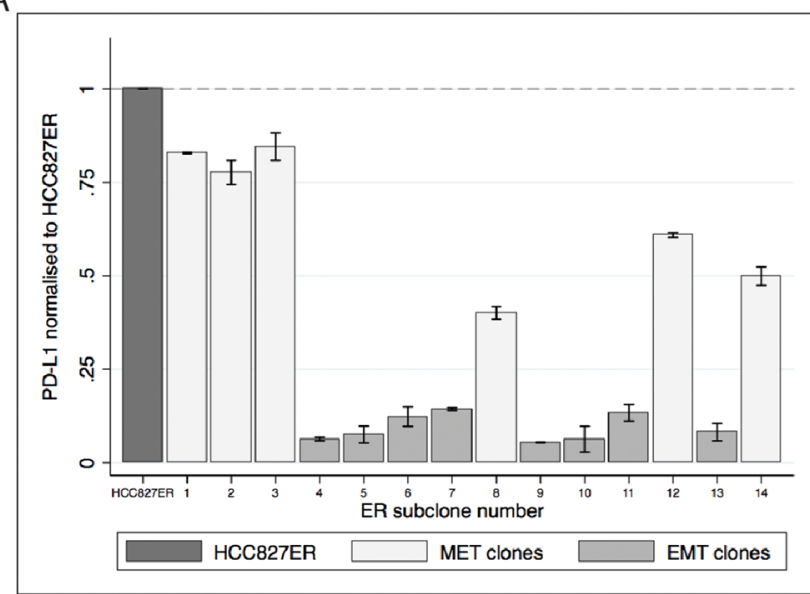

C

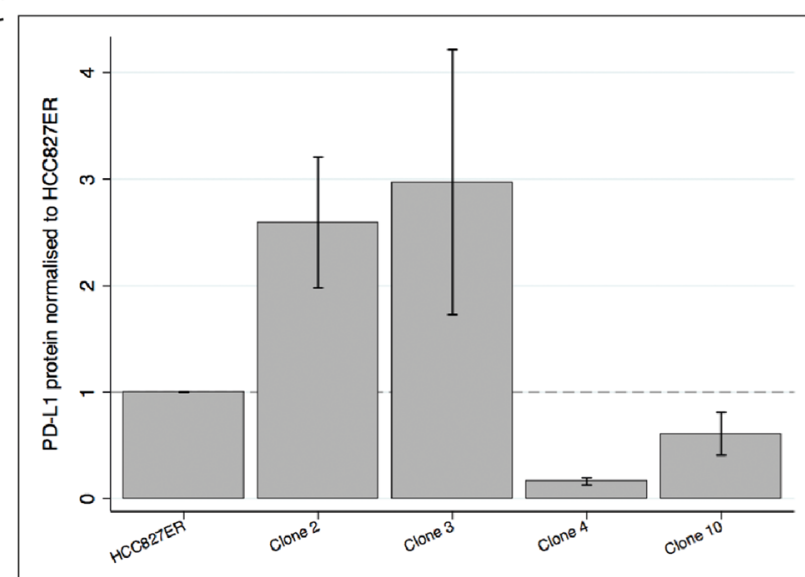

B

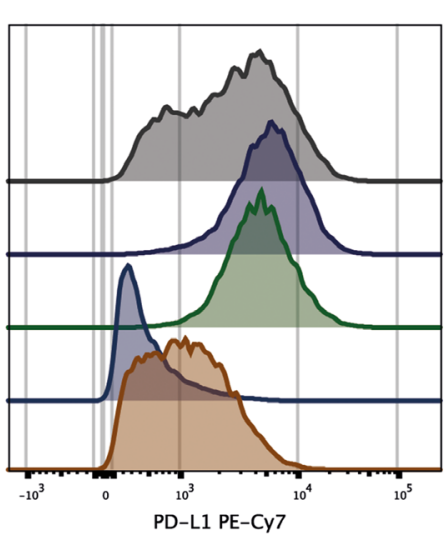

\begin{tabular}{|l|l|l|}
\hline & Sample name & MFI \\
\hline & Untreated control & 3040 \\
\hline \hline & Clone 2 (MET) & 5725 \\
\hline \hline & Clone 3 (MET) & 4711 \\
\hline \hline & Clone 4 (EMT) & 409 \\
\hline \hline & Clone 10 (EMT) & 1152 \\
\hline
\end{tabular}

Figure 3: PD-L1 gene and protein expression in HCC827ER sub clones. (A) $P D-L 1$ gene expression in the 14 clones (normalized to HCC827ER). Expression of $P D-L 1$ is markedly higher in the MET clones compared to the EMT clones. (B) Representable histogram from a flow cytometry analysis of HCC827ER and four clones. MFI values are found in the table. (C) A bar graph showing the mean and $\mathrm{SD}$ of PD-L1 protein expression collected from three individual experiments. 
by activation of the MAPK or PI3K/Akt pathways (bypass mechanisms) extraordinarily interesting in regard to immunotherapy, both when it comes to second line treatment of patients and the use of PD-L1 as biomarker of response. Jiang and coworkers have already presented data showing that BRAF inhibitor resistant melanoma cell lines increase the expression of PD-L1 using the PI3KSTAT3 pathway [27]. Further, a study comparing tumor PD-L1 expression in biopsies taken before initiation of gefitinib and at resistance found increased expression in 7 of 18 patients [20]. They also found that the increase in PD-L1 expression was associated with MET positivity (as measured by immunohistochemistry). The authors note that the number of patients included in this study is too low to make general conclusions, though in the context of ours and other studies, the biological evidence seems to back this clinical finding. However, for full disclosure on the biological mechanism, further studies are needed. In vitro RNAi studies and/or in vivo models should be used to investigate expression of PD-L1 in MET knockout models to present evidence of MET-regulated PDL1 expression. Furthermore, the biological effect of the increase in PD-L1 expression should be investigated in the presence of a functional immune system.

The erlotinib-resistant cell line investigated in the present study consists of subclones that can be divided into MET-amplified and EMT clones [21]. When investigating the clones separately we found that the $M E T$-amplified clones have a markedly higher expression of PD-L1 than does the EMT clones. Lack of induced PD-L1 expression in EMT clones contradicts previous research on the subject. Generally studies have shown that the mesenchymal phenotype (intrinsic or induced by EMT) is associated with increased PD-L1 expression in different cancers [28-32]. In lung cancer specifically, Chen and colleagues have shown that PD-L1 expression is regulated by the miR-200/ZEB1 loop, and suggest that increased expression of ZEB1 in mesenchymal cell lines prompt increased expression of PD-L1. Despite increased ZEB1 expression in our resistant EMT clones [21], we did not observe increased PD-L1 expression. Further, Kurimoto and colleagues find that induction of EMT in HCC827 results in increased expression of PD-L1 [29]. A possibility could be that induction of EMT by TGF- $\beta$ and FGF2 used in that study, results in a different phenotype than induction by acquired erlotinib resistance.

The data presented here suggest that erlotinibresistant cells increase the expression of PD-L1 through a mechanism involving MET and MAPK activation. Treatment with MET inhibitors in relevant cases could possibly prevent PD-L1 expression and thereby immune evasion. Furthermore, the PD-L1 fluctuations observed here, urges clinicians to exercise caution when evaluating the use of immunotherapy after TKI-treatment, based on PD-L1 measurements on biopsies taken at the time of diagnosis.

\section{MATERIALS AND METHODS}

\section{Cell lines and treatment}

The cell line HCC827 was purchased from ATCC. An erlotinib-resistant HCC827 cell line (HCC827ER) was generated as previously described [21]. Further, HCC827ER clones were established using minimal dilution [21]. All cells were maintained in RPMI 1640 media supplemented with $1 \%$ Penicillin-Streptomycin (Gibco), 1\% Hepes $1 \mathrm{M}$ buffer solution (Gibco), 1\% Sodium Pyruvate (Gibco), 10\% Fetal Bovine Serum (Gibco), and 1\% $250 \mu \mathrm{g} / \mathrm{mL}$ Amphotericin B solution (Sigma-Aldrich). Further, the resistant cell line and clones were maintained in $5 \mu \mathrm{M}$ erlotinib (Selleckchem).

For all mRNA and flow cytometry experiments cells were plated in 6-well plates and incubated for $24 \mathrm{~h}$ (300,000 cells/well). After incubation the cells were treated with drug and incubated for $72 \mathrm{~h}$ prior to harvest.

The inhibitors erlotinib, crizotinib, and SCH772984 were all purchased from Selleckchem.

\section{Viability}

For cell viability studies cells were plated in 96-well plates (5000 cells/well) and incubated for $24 \mathrm{~h}$. Cells were treated with drug as indicated and incubated for $72 \mathrm{~h}$ prior to viability assessment. Cell viability was tested using an MTS assay (CellTiter $96^{\circledR}$ AQueous Non-Radioactive Cell Proliferation Assay, Promega) following the manufacturer's instructions. Colorimetric measurement was performed using a Multiscan Ascent plate reader (Thermo Electron Corporation).

\section{Western blotting}

Protein was harvested using Lysis Buffer 17 (R\&D Systems) supplemented with $10 \mu \mathrm{g} / \mathrm{mL}$ of each inhibitor Pepstatin, Leupeptin, and Aprotinin. Harvest was performed according to manufacturer's protocol. The protein concentration was determined using the Qubit ${ }^{\circledR}$ 2.0 Fluorometer (Thermo Scientific). $30 \mu \mathrm{g}$ protein was resolved on $4-12 \%$ Bis-Tris gels (Life Technologies) and transferred to a PVDF membrane (Thermo Scientific). Membranes were blocked in 1X TBST with 5\% non-fat dry milk (protein-specific antibodies) or 5\% BSA (phosphospecific antibodies). For detection the following primary antibodies and dilutions were used: anti-EGFR (Abcam, 1:1000), anti-EGFR phospho-Tyr1173 (LSBio, 1:500), antiAkt (Cell Signaling Technology, 1:500), anti-Akt phosphoSer473 (Cell Signaling Technology, 1:500), anti-MAPK (Cell Signaling Technology, 1:1000), anti-MAPK phosphoThr202/Tyr204 (Cell Signaling Technology, 1:1000), and anti-Histone H3 (Cell Signaling Technology, 1:2000). Antibodies were diluted in either 1X TBST with 5\% non-fat dry milk or $5 \%$ BSA corresponding to the blocking agent. 
Goat anti-mouse (DAKO, 1:4000) and goat anti-rabbit secondary antibody (Cell Signaling Technology, 1:5000) were diluted in $1 \mathrm{X}$ TBST with 5\% non-fat dry milk and used for detection of total-MET and the remaining proteins, respectively. The membranes were added SuperSignal Dura West Chemiluminescent Substrate (ECL) (Thermo Scientific) followed by development in an ImageQuant LAS 4000 scanner (GE Healthcare Life Sciences).

\section{cDNA synthesis and quantitative PCR}

For RNA analysis cells were harvested using Nunc Cell Scrapers (Thermo Scientific), centrifuged, and resuspended in $350 \mu \mathrm{L}$ RLT buffer (Qiagen). Total RNA was extracted using RNeasy mini kit on a QIACube instrument (Qiagen). RNA concentration was determined using the NanoDrop 2000 (Thermo Fisher Scientific). $200 \mathrm{ng}$ RNA was used for cDNA synthesis. cDNA was synthesised in a $20 \mu \mathrm{L}$ reaction consisting of $2.5 \mu \mathrm{M}$ Oligo(dT) (DNA Technology), $1 \mathrm{mM}$ of each dNTP (VWR), 2.5 units/ $\mu \mathrm{L}$ MulV Reverse Transcriptase, 1 units/ $\mu \mathrm{L}$ RNase inhibitors, 1xPCR buffer, and $6.25 \mathrm{mM} \mathrm{MgCL2}$ (all from Applied Biosystems). Quantitative PCR (qPCR) was performed using LightCycler 480 SYBR Green I Master on the LightCycler 480 platform (Roche).

Five reference genes ( $\beta$-actin, HMBS, GAPDH, YWHAZ and B2M) were tested and compared using the Normfinder Software [33]. Among these $\beta$-actin (ACTB) was found most stably expressed in the cell lines and was used as reference gene for the following experiments.

For the experiments presented the following primer sequences, concentrations and annealing temperatures were applied:

ACTB: forward 5'- GGCGCCACCACCATGTA CCCT-3', reverse 5'- AGGGGCCGGACTCGTCATACT$3^{\prime}, 0.25 \mu \mathrm{M}, 68^{\circ} \mathrm{C}$.

PD-L1: forward 5'- GGTGGTGCCGACTACAA GCGA-3', reverse 5'-TGACTTCGGCCTTGGGGTAGC$3^{\prime}, 0.25 \mu \mathrm{M}, 64^{\circ} \mathrm{C}$.

MET: forward 5'- TGGAGACACTGGATGGGAGT3', reverse 5'-CAGCGCGTTGACTTATTCAT-3', $0.25 \mu \mathrm{M}$, $60^{\circ} \mathrm{C}$.

All primers were purchased from Eurofins Genomics. Gene expression was measured and calculated using the Lightcycler 480 instrument (Roche) and the second derivative max method using standard curves.

All experiments were performed in biological triplicates, except from the analysis of RNA from the resistance development (Figure 1A and 1B) and from the 14 sub-clones (Figure 3A), where only one specimen was available. All gene expression analyses were performed in technical triplicates.

\section{Flow cytometry}

Cells were detached from wells using PBS $/ 0.5 \%$ $\mathrm{BSA} / 2 \mathrm{mM}$ EDTA, washed once in staining buffer
(PBS/0.5\% BSA/0.09\% sodium azide), and kept on ice. Non-specific antibody binding was blocked with $10 \%$ mouse serum (from healthy C57BL/6J mice) for $15 \mathrm{~min}$ at $4^{\circ} \mathrm{C}$. Samples were then stained with Live/Dead fixable dye near-IR (Life Technologies) and mouse anti-human PD-L1 PE-Cy7 (0.5 g/mL, clone 29E.2A3, BioLegend) in stain buffer for $30 \mathrm{~min}$ (in the dark at $4^{\circ} \mathrm{C}$ ). Cells were washed in stain buffer, fixed in PBS $/ 0.9 \%$ formaldehyde (Sigma-Aldrich), and analysed immediately on an LSR Fortessa flow cytometer (BD Biosciences).

Compensation was done using single stained beads, for PD-L1 PE-Cy7 Comp Beads Plus (BD Biosciences) were used and for Live/Dead nIR ArC Amine Reactive Compensation beads (Life Technologies) were used. Data was analysed and figures were made using FlowJo 10.0.7 for Mac (FlowJo, LLC). For gating strategy, see Supplementary Figure 1.

All flow cytometry experiments were performed using the LSR Fortessa flow cytometer at the FACS Core Facility, Aarhus University, Denmark.

\section{Graphs and statistics}

All graphs and statistics are produced in Stata 13 (StataCorp, 2013).

Gene and protein expression data from TKI experiments is collected from three individual setups. In each experiment data was normalised to an untreated control, and the mean of this normalised data is presented in graphs.

All data is presented as mean \pm standard deviation (SD) (mean of biological triplicates). The two-tailed Student's $t$-test was used to assess the difference between two groups, and $p$-values $<0.05$ were considered significant.

\section{ACKNOWLEDGMENTS AND FUNDING}

The authors wish to acknowledge Birgit Westh Mortensen for technical assistance.

\section{CONFLICTS OF INTEREST}

The authors have no conflicts of interest to declare.

\section{REFERENCES}

1. Pao W, Girard N. New driver mutations in non-small-cell lung cancer. Lancet Oncol. 2011; 12:175-80. https://doi. org/10.1016/S1470-2045(10)70087-5.

2. Petrelli F, Borgonovo K, Cabiddu M, Barni S. Efficacy of EGFR Tyrosine Kinase Inhibitors in Patients With EGFRMutated Non-Small-Cell Lung Cancer: A Meta-Analysis of 13 Randomized Trials. Clin Lung Cancer. 2012; 13:107-14. https://doi.org/10.1016/j.cllc.2011.08.005.

3. Oxnard GR, Arcila ME, Chmielecki J, Ladanyi M, Miller VA, Pao W. New Strategies in Overcoming Acquired 
Resistance to Epidermal Growth Factor Receptor Tyrosine Kinase Inhibitors in Lung Cancer. Clin Cancer Res. 2011; 17 : 5530-7. https://doi.org/10.1158/1078-0432.CCR-10-2571.

4. Camidge DR, Pao W, Sequist LV. Acquired resistance to TKIs in solid tumours: learning from lung cancer. Nat Rev Clin Oncol. 2014; 11:473-81. https://doi.org/10.1038/ nrclinonc.2014.104.

5. Pardoll DM. The blockade of immune checkpoints in cancer immunotherapy. Nat Rev Cancer. 2012; 12:252-64. https:// doi.org/10.1038/nrc3239.

6. Dong H, Strome SE, Salomao DR, Tamura H, Hirano F, Flies DB, Roche PC, Lu J, Zhu G, Tamada K, Lennon VA, Celis E, Chen L. Tumor-associated B7-H1 promotes T-cell apoptosis: A potential mechanism of immune evasion. Nat Med. 2002; 8:793-800. https://doi.org/10.1038/nm730.

7. Iwai Y, Ishida M, Tanaka Y, Okazaki T, Honjo T, Minato N. Involvement of PD-L1 on tumor cells in the escape from host immune system and tumor immunotherapy by PD-L1 blockade. Proc Natl Acad Sci USA. 2002; 99:12293-7. https://doi.org/10.1073/pnas.192461099.

8. Blank C, Brown I, Peterson AC, Spiotto M, Iwai Y, Honjo T, Gajewski TF. PD-L1/B7H-1 inhibits the effector phase of tumor rejection by $\mathrm{T}$ cell receptor (TCR) transgenic CD8+ T cells. Cancer Res. 2004; 64:1140-5.

9. Langer CJ. Emerging immunotherapies in the treatment of non-small cell lung cancer (NSCLC): the role of immune checkpoint inhibitors. Am J Clin Oncol. 2015; 38:422-30. https://doi.org/10.1097/COC.0000000000000059.

10. Garon EB, Rizvi NA, Hui R, Leighl N, Balmanoukian AS, Eder JP, Patnaik A, Aggarwal C, Gubens M, Horn L, Carcereny E, Ahn MJ, Felip E, et al, and KEYNOTE-001 Investigators. Pembrolizumab for the treatment of nonsmall-cell lung cancer. N Engl J Med. 2015; 372:2018-28. https://doi.org/10.1056/NEJMoa1501824.

11. Topalian SL, Hodi FS, Brahmer JR, Gettinger SN, Smith DC, McDermott DF, Powderly JD, Carvajal RD, Sosman JA, Atkins MB, Leming PD, Spigel DR, Antonia SJ, et al. Safety, activity, and immune correlates of anti-PD-1 antibody in cancer. N Engl J Med. 2012; 366:2443-54. https://doi.org/10.1056/NEJMoa1200690.

12. Azuma K, Ota K, Kawahara A, Hattori S, Iwama E, Harada T, Matsumoto K, Takayama K, Takamori S, Kage M, Hoshino T, Nakanishi Y, Okamoto I. Association of PD-L1 overexpression with activating EGFR mutations in surgically resected nonsmall-cell lung cancer. Ann Oncol. 2014; 25:1935-40. https://doi.org/10.1093/annonc/ mdu242.

13. D'Incecco A, Andreozzi M, Ludovini V, Rossi E, Capodanno A, Landi L, Tibaldi C, Minuti G, Salvini J, Coppi E, Chella A, Fontanini G, Filice ME, et al. PD-1 and PD-L1 expression in molecularly selected non-small-cell lung cancer patients. Ann Oncol. 2015; 112:95-102. https:// doi.org/10.1038/bjc.2014.555.

14. Okita R, Maeda A, Shimizu K, Nojima Y, Saisho S, Nakata M. PD-L1 overexpression is partially regulated by EGFR/HER2 signaling and associated with poor prognosis in patients with non-small-cell lung cancer. Cancer Immunol Immunother. 2017; 66:865-76. https:// doi.org/10.1007/s00262-017-1986-y.

15. Yang CY, Lin MW, Chang YL, Wu CT, Yang PC. Programmed cell death-ligand 1 expression in surgically resected stage I pulmonary adenocarcinoma and its correlation with driver mutations and clinical outcomes. Eur J Cancer. 2014; 50:1361-9. https://doi.org/10.1016/j. ejca.2014.01.018.

16. Chen N, Fang W, Zhan J, Hong S, Tang Y, Kang S, Zhang Y, He X, Zhou T, Qin T, Huang Y, Yi X, Zhang L. Upregulation of PD-L1 by EGFR Activation Mediates the Immune Escape in EGFR-Driven NSCLC: Implication for Optional Immune Targeted Therapy for NSCLC Patients with EGFR Mutation. J Thorac Oncol. 2015; 10:910-23. https://doi.org/10.1097/JTO.0000000000000500.

17. Lin K, Cheng J, Yang T, Li Y, Zhu B. EGFR-TKI downregulates $\mathrm{PD}-\mathrm{L} 1$ in EGFR mutant NSCLC through

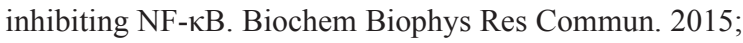
463:95-101. https://doi.org/10.1016/j.bbrc.2015.05.030.

18. Sumimoto H, Takano A, Teramoto K, Daigo Y. RASMitogen-Activated Protein Kinase Signal Is Required for Enhanced PD-L1 Expression in Human Lung Cancers. PLoS One. 2016; 11:e0166626. https://doi.org/10.1371/ journal.pone.0166626.

19. Akbay EA, Koyama S, Carretero J, Altabef A, Tchaicha JH, Christensen CL, Mikse OR, Cherniack AD, Beauchamp EM, Pugh TJ, Wilkerson MD, Fecci PE, Butaney M, et al. Activation of the PD-1 Pathway Contributes to Immune Escape in EGFR-Driven Lung Tumors. Cancer Discov. 2013; 3:1355-63. https://doi.org/10.1158/2159-8290.CD-13-0310.

20. Han JJ, Kim DW, Koh J, Keam B, Kim TM, Jeon YK, Lee SH, Chung DH, Heo DS. Change in PD-L1 Expression After Acquiring Resistance to Gefitinib in EGFR-Mutant Non-Small-Cell Lung Cancer. Clin Lung Cancer. 2016; 17:263-270.e2. https://doi.org/10.1016/j.cllc.2015.11.006.

21. Jakobsen KR, Demuth C, Madsen AT, Hussmann D, VadNielsen J, Nielsen AL, Sorensen BS. MET amplification and epithelial-to-mesenchymal transition exist as parallel resistance mechanisms in erlotinib-resistant, EGFRmutated, NSCLC HCC827 cells. Oncogenesis. 2017; 6:e307. https://doi.org/10.1038/oncsis.2017.17.

22. Ota K, Azuma K, Kawahara A, Hattori S, Iwama E, Tanizaki J, Harada T, Matsumoto K, Takayama K, Takamori S, Kage M, Hoshino T, Nakanishi Y, et al. Induction of PD-L1 Expression by the EML4-ALK Oncoprotein and Downstream Signaling Pathways in Non-Small Cell Lung Cancer. Clin Cancer Res. 2015; 21:4014-21. https://doi. org/10.1158/1078-0432.CCR-15-0016.

23. Hanahan D, Weinberg RA. Hallmarks of Cancer: The Next Generation. Cell. 2011; 144:646-74. https://doi. org/10.1016/j.cell.2011.02.013.

24. Costa DB, Kobayashi S, Yeo WL, Hamada A. Serum Concentrations of Erlotinib at a Dose of $25 \mathrm{mg}$ Daily. J 
Thorac Oncol. 2010; 5:1311-2. https://doi.org/10.1097/ JTO.0b013e3181edf55c.

25. Yamamoto R, Nishikori M, Tashima M, Sakai T, Ichinohe T, Takaori-Kondo A, Ohmori K, Uchiyama T. B7-H1 expression is regulated by MEK/ERK signaling pathway in anaplastic large cell lymphoma and Hodgkin lymphoma. Cancer Sci. 2009; 100:2093-100. https://doi.org/10.1111/ j.1349-7006.2009.01302.x.

26. Lastwika KJ, Wilson W, Li QK, Norris J, Xu H, Ghazarian SR, Kitagawa H, Kawabata S, Taube JM, Yao S, Liu LN, Gills JJ, Dennis PA. Control of PD-L1 Expression by Oncogenic Activation of the AKT-mTOR Pathway in Non-Small Cell Lung Cancer. Cancer Res. 2016; 76:22738. https://doi.org/10.1158/0008-5472.CAN-14-3362.

27. Jiang X, Zhou J, Giobbie-Hurder A, Wargo J, Hodi FS. The activation of MAPK in melanoma cells resistant to BRAF inhibition promotes PD-L1 expression that is reversible by MEK and PI3K inhibition. Clin Cancer Res. 2013; 19:598-609. https://doi.org/10.1158/1078-0432.CCR-122731.

28. Chen L, Gibbons DL, Goswami S, Cortez MA, Ahn YH, Byers LA, Zhang X, Yi X, Dwyer D, Lin W, Diao L, Wang J, Roybal JD, et al. Metastasis is regulated via microRNA-200/ZEB1 axis control of tumour cell PDL1 expression and intratumoral immunosuppression. Nat Commun. 2014; 5:5241. https://doi.org/10.1038/ ncomms6241.

29. Kurimoto R, Iwasawa S, Ebata T, Ishiwata T, Sekine I, Tada Y, Tatsumi K, Koide S, Iwama A, Takiguchi Y. Drug resistance originating from a TGF- $\beta /$ FGF-2-driven epithelial-to-mesenchymal transition and its reversion in human lung adenocarcinoma cell lines harboring an EGFR mutation. Int J Oncol. 2016; 48:1825-36. https://doi. org/10.3892/ijo.2016.3419.

30. Mak MP, Tong P, Diao L, Cardnell RJ, Gibbons DL, William WN, Skoulidis F, Parra ER, Rodriguez-Canales J, Wistuba II, Heymach JV, Weinstein JN, Coombes KR, et al. A Patient-Derived, Pan-Cancer EMT Signature Identifies Global Molecular Alterations and Immune Target Enrichment Following Epithelial-to-Mesenchymal Transition. Clin Cancer Res. 2016; 22:609-20. https://doi. org/10.1158/1078-0432.CCR-15-0876.

31. Ock CY, Kim S, Keam B, Kim M, Kim TM, Kim JH, Jeon YK, Lee JS, Kwon SK, Hah JH, Kwon TK, Kim DW, Wu HG, et al. PD-L1 expression is associated with epithelial-mesenchymal transition in head and neck squamous cell carcinoma. Oncotarget. 2016; 7:15901-14. https://doi.org/10.18632/oncotarget.7431.

32. Kim S, Koh J, Kim MY, Kwon D, Go H, Kim YA, Jeon YK, Chung DH. PD-L1 expression is associated with epithelialto-mesenchymal transition in adenocarcinoma of the lung. Hum Pathol. 2016; 58:7-14. https://doi.org/10.1016/j. humpath.2016.07.007.

33. Andersen CL, Jensen JL, Ørntoft TF. Normalization of real-time quantitative reverse transcription-PCR data: a model-based variance estimation approach to identify genes suited for normalization, applied to bladder and colon cancer data sets. Cancer Res. 2004; 64:5245-50. https://doi. org/10.1158/0008-5472.CAN-04-0496. 